\title{
Optimal Consumption and Investment with Lévy Processes*
}

\author{
José Fajardo Barbachan ${ }^{* *}$
}

Summary: 1. Introduction; 2. Model; 3. Lévy processes and equivalent martingale measures; 4. Optimization problem; 5. Choosing a measure; 6. Example; 7. Conclusions.

Keywords: Lévy processes; incomplete markets.

JEL Codes: G11; C61.

We study the intertemporal consumption and investment problem in a continuous time setting when the security prices follow a Geometric Lévy process. Using stochastic calculus for semimartingales we obtain conditions for the existence of optimal consumption policies. Also, we give a charaterization of the equivalent martingale measures.

Estudamos o problema do consumo e investimento intertemporal em tempo contínuo, quando os preços dos ativos seguem um processo de Lévy Geométrico. Usando cálculo estócastico para semimartingalas obtemos condições para a existência de políticas ótimas de consumo. Também, mostramos a caracterização das medidas martingalas equivalentes.

\section{Introduction}

The intertemporal optimal consumption and investment problem in a continuous time setting is a important task in the finance literature. The first to study this problem was Merton (1971), using stochastic dynamic programming he obtained explicit solutions for an economy with incomplete markets in which security prices follow a geometric Brownian motion, the endowments follow a Poisson process and the investor has a negative exponential utility with an infinite horizon.

Many other authors have tried to improve the above model adding income stream, transaction costs, borrowing constraints and other facts that make the

\footnotetext{
${ }^{*}$ This paper was received in Nov. 2002 and approved in Feb. 2003. I want to thank Maria Eulália Vares for valuable comments and IMPA-Brazil, where this project was partially developed. I also acknowledge financial support from CNPq, Brazil.

** IBMEC Business School. E-mail: pepe@ibmecrj.br
} 
model more real. In all these models the log-Normal hypothesis or a slight modification of it is assumed for stock returns. Unfortunately, it is very well known that this hypothesis did not hold for the majority of stocks, since they present stylized facts like: fat tails, asymmetry, autocorrelation, etc. For a survey about the principal results on stylized facts see Rydberg (1997).

For this reason, recently some results, assuming more general process to model stock returns, have appear in the literature. Kramkov and Schachermayer (1999) prove that if the asymptotic elasticity of the utility is strictly less than one, then there is a solution for the optimal consumption and investment problem.

Benth et al. (2001) have obtained a solution for the optimal consumptioninvestment problem assuming that the dynamic programming principle holds and that the stock returns are modelled by a pure-jump Lévy processes, to solve this problem they used a viscosity solution approach. Fajardo (2000) proves that when the stock returns follow a Hyperbolic Lévy motion and we choose a particular equivalent martingale measure (EMM) to construct the state price density, then it is possible to find conditions on the wealth process and strategies that guarantee the existence of optimal policies.

In this paper we extend the latter result considering a huge class of processes called geometric Lévy processes and all the set of EMM. To this end we characterize all the EMM that exist in the market. The paper is organized as follows: in section 2 we describe the model, in section 3 we address the existence of EMM in a Lévy market, that is, when stock prices follow a Geometric Lévy Motion. In section 4 we introduce the optimal problem and state the main result. In section 5 we show how to choose an EMM and in the last sections we present an example and the conclusions.

\section{Model}

We will consider a financial market $\mathfrak{M}$ consisting of 2 assets. The first is called bond (the riskless asset) and the second is called stock (the risky asset). We denote by $B(t)$ and $P(t)$ the bond's and stock's price at each time $t \in[0, T]$, respectively. The evolution of these prices are modelled by the following equations:

$$
\begin{gathered}
d B(t)=r(t) B(t) d t, \quad B(0)=1 \\
d P(t)=P\left(t^{-}\right)\left[\rho_{t} d t+\sigma_{t} d Y(t)\right], \quad P(0) \in(0, \infty)
\end{gathered}
$$


In this model the sources of risk are modelled by a Lévy process $Y(t), 0 \leq t \leq T$, i.e., a process with independent and stationary increments. $Y$ is defined on a given complete probability space $(\Omega, \mathcal{F}, \mathbf{P})$ and denote by $\mathbf{F}=\{\mathcal{F}(\mathbf{t}), \mathbf{0} \leq \mathbf{t} \leq \mathbf{T}\}$ the $P$ - augmentation ${ }^{1}$ of the natural filtration generated by $Y$ :

$$
\mathcal{F}_{Y}(t)=\sigma(Y(s), 0 \leq s \leq t), 0 \leq t \leq T
$$

time horizon will be considered finite. The positiveness of the stock price will be analyzed in the next section. The interest rate $\{r(t): 0 \leq t \leq T\}$, assumed finite, the appreciation rate $\{\rho(t), 0 \leq t \leq T\}$, and the volatilities $\sigma(t), 0 \leq t \leq T$ will be referred as the coefficients of the financial market $\mathfrak{M}$. We assume that these coefficients are deterministic continuous functions. Now we introduce a small investor (his decisions does not affect the market prices), who will decide at each moment $t \in[0, T]$ :

1. How much money $\pi(t)$ he wants to invest in the stock;

2. His cumulative consumption $C(t)$.

Of course, these decisions must be made without foreknowledge of future events, so $C$ and $\pi$ must be adapted processes. ${ }^{2}$ If we denote by $X(t)$ the agent's wealth at time $t$, then the amount invested in the bond will be $X(t)-\pi(t)$. From here, (1) and (2), we obtain the following equation for the wealth:

$$
\begin{aligned}
d X(t) & =\pi(t) \frac{d P(t)}{P\left(t^{-}\right)}+(X(t)-\pi(t)) \frac{d B(t)}{B(t)}-d C(t) \\
& =\pi(t)[\rho(t) d t+\sigma(t) d Y(t)]+(X(t)-\pi(t)) r(t) d t-d C(t) \\
= & r(t) X(t) d t+\pi(t)[(\rho(t)-r(t)) d t+\sigma(t) d Y(t)]-d C(t)
\end{aligned}
$$

The solution of this linear stochastic differential equation with initial condition $x \in \mathbb{R}$, i.e. $X(0)=x$ is:

\footnotetext{
${ }^{1}$ The augmented filtration $\mathbf{F}$ is defined by $\mathcal{F}(t)=\sigma\left(\mathcal{F}_{Y}(t) \cup \mathcal{N}\right)$, where $\mathcal{N}=$ $\{E \subset \Omega: \exists G \in \mathcal{F}$ with $E \subseteq G, \mathbf{P}(\mathbf{G})=\mathbf{0}\}$ denotes the set of $\mathbf{P}$ - null events.

${ }^{2}$ We said that a process $\left\{X_{t}\right\}$ is adapted with respect to $\mathbf{F}$ if for all $t \in[0, T], X_{t}$ is an $\mathcal{F}(t)$-measurable random variable.
} 


$$
\gamma(t) X(t)=x-\int_{0}^{t} \gamma(s) d C(s)+\int_{0}^{t} \gamma(s) \pi(s)[\sigma(s) d Y(s)+(\rho(s)-r(s)) d s]
$$

for all $0 \leq t \leq T$. Where

$$
\gamma(t) \triangleq e^{-\int_{0}^{t} r(s) d s}
$$

is the discount factor in $\mathfrak{M}$. Now we give some definitions:

\section{Definition 1}

(i) An $\mathbf{F}$ - adapted process $C=C(t), 0 \leq t \leq T$ with nondecreasing, right-continuous paths, left-hand limits and $C(0)=0, C(T)<\infty$ a.s is called a cumulative consumption process.

(ii) An $\mathbf{F}$ - progressively measurable, càdlàg, $\mathbb{R}$ - valued process $\pi=\{\pi(t), 0 \leq t \leq T\}$ with

$$
\int_{0}^{T}|\pi(t) \sigma(t)|^{2} d t+\int_{0}^{T}|\pi(t)(\rho(t)-r(t))| d t<\infty, a . s
$$

is called a portfolio process.

(iii) For a given $x \in \mathbb{R}$ and $(\pi, C)$ as above, the process $X(t)=X^{x, \pi, C}(t)$ of (3), (5) is called the wealth process corresponding to initial capital $x$, portfolio $\pi$, and cumulative consumption process $C$.

It is important to notice that $(X(t)-\pi(t))$ and $\pi(t)$ can take negative values, in other words short-sales of stock and borrowing at interest rate $r(\cdot)$ are allowed. So we need to impose some restriction on portfolios.

Definition 2: We say that a given portfolio process $\pi(\cdot)$ is tame, if the associated discounted gain process: 


$$
M^{\pi}(t) \triangleq \int_{0}^{t} \gamma(s) \pi(s)[\sigma(s) d Y(s)+(\rho(s)-r(s)) d s]
$$

is a.s. bounded from below by some real constant:

$$
\mathbf{P}\left[M^{\pi}(t) \geq q_{\pi}, \forall 0 \leq t \leq T\right]=1 \text { for some } q_{\pi} \in \mathbb{R}
$$

Condition (9) is necessary to avoid doubling strategies, that is, portfolios that attain arbitrary large values of wealth with probability one at $t=T$, starting with zero initial capital at $t=0 .^{3}$

Definition 3 A tame portfolio that satisfies:

$$
\mathbf{P}\left[M^{\pi}(T) \geq 0\right]=1, \quad \mathbf{P}\left[M^{\pi}(T)>0\right]>0
$$

is called an "arbitrage opportunity" (or free lunch). We say that a market Mis arbitrage free if no such portfolio exist.

The free lunch interpretation of (10) is clear: starting with zero initial capital and using the strategy $\pi(\cdot)$ at the end of the period $t=T$. Since $\gamma(T)=B(T)^{-1}$, we have $X(T)=X^{0, \pi, 0}(T)=B(T) M^{\pi}(T)$, then $X(T) \geq 0$ a.s, i.e. no risk and positive probability of gain $\mathbf{P}[X(T)>0]>0$.

So we need conditions for precluding these arbitrage opportunities. We know that the existence of an EMM in general context rule out this opportunities. In our context Eberlein and Jacod (1997) show that when $r(t)=r, \forall t \in[0, T]$, there would be EMM and Chan (1999) shows analogous result with $r(\cdot)$ being a deterministic continuous function. We will show that these existence imply that our market is arbitrage free. An important consequence of our extension is that many market models are incomplete then we have not an unique EMM. The criterion to choose one of these EMM will be also presented.

\section{Lévy Processes and Equivalent Martingale Measures}

In this section we characterize all the equivalent martingale measures in the model introduced in section 2 . We know that all the infinitely divisible distribu-

\footnotetext{
${ }^{3}$ See Karatzas and Shreve (1998) for an example.
} 
tions $^{4}\left(Y_{t}\right)$ admit the following Lévy-Khintchine representation:

$$
\phi(u)=\exp \left(i a u-\frac{c}{2} u^{2}+\int\left(e^{i u x}-1-i u x \mathbf{1}_{[|\mathbf{x}| \leq \mathbf{1}]}\right) G(d x)\right)
$$

where $\phi$ is the characteristic function of the infinitely divisible distribution, $a$ is the drift, $c$ is the quadratic variation coefficient and $G$ is a positive measure with $\int \min x^{2}, 1 G(d x)<\infty$. This measure is called the Lévy measure and describes the jumps of the process.

We also know that all Lévy process must be a linear combination of a standard Brownian Motion $\left(W_{t}\right)$ and a quadratic pure jump process ${ }^{5}\left(N_{t}\right)$ which is independent of the Brownian Motion $W_{t}$, then

$$
Y_{t}=c W_{t}+N_{t}
$$

The process $N_{t}$ has a Lévy decomposition: Let $L(d t, d x)$ be a Poisson measure on $\mathbb{R}^{+} \times \mathbb{R} \backslash 0$ with expectation (or compensator) measure $d t \times G^{6}$, then:

$$
\begin{aligned}
N_{t} & =\int_{[|x|<1]} x(L((0, t], d x)-t G(d x))+\int_{[|x| \geq 1]} x L((0, t], d x) \\
& +t E\left[N_{1}-\int_{|x| \geq 1} x G(d x)\right]
\end{aligned}
$$

Now assume that ${ }^{7}$

$$
E\left[\exp \left(-b Y_{1}\right)\right]<\infty \forall b \in\left(-b_{1}, b_{2}\right)
$$

and

$$
\int_{[|x| \geq 1]} e^{-b x} d G(x)<\infty \forall b \in\left(-b_{1}, b_{2}\right)
$$

where $0<b_{1}, b_{2} \leq \infty$. The first assumption said that $Y_{t}$ has all moments finite and the second is technical and will let us separate integrands. With this in mind we can return to the jumps and transform the equation (12) into:

\footnotetext{
${ }^{4}$ An infinite divisible distribution generates a Lévy processes, for abuse of notation we use $Y_{t}$ for both. For more details see Sato (1999) or Shiryaev (1999).

${ }^{5} \mathrm{~A}$ process $X$ is said to be a quadratic pure jump process if $\langle N\rangle^{c} \equiv 0$, where $\langle N\rangle^{c}$ is the continuous part of its quadratic variation $\langle N\rangle$. Remember that $\langle N\rangle$ is the process such that $\left(N_{t}\right)^{2}-\langle N\rangle_{t}$ is a martingale.

${ }^{6} \forall B \in \mathbb{R}^{+} \times \mathbb{R} \backslash 0, L(B)$ has Poisson distribution with parameter $(d t \times G)(B)$

${ }^{7} E(\cdot)$ denote the expectation with respect to $\mathbf{P}$
} 


$$
N_{t}=\int_{\mathbb{R}} x(L((0, t], d x)-t G(d x))+t E N_{1}
$$

It is easy to see that the process

$$
M_{t}=\int_{\mathbb{R}} x(L((0, t], d x)-t G(d x))
$$

is a martingale. Then $N_{t}=M_{t}+a t$, with $a=E N_{1}$, as a consequence the original process can be written as

$$
Y_{t}=M_{t}+c W_{t}+a t
$$

Before passing to characterize the absolutely continuous measures with respect to the original measure, let us introduce some elements of stochastic calculus: for any measurable function $f(t, x)$ we have

$$
\sum_{0<s \leq t} f\left(s, \Delta N_{s}\right)=\int_{0}^{t} \int_{\mathbb{R}} f(s, x) L(d s, d x)
$$

and for any $C^{2}$ function $f$, we have the Generalized Itô's formula for càdlàg semimartingales $X^{1}, \ldots, X^{n}$ :

$$
\begin{aligned}
d f\left(X_{t}^{1}, . ., X_{t}^{n}\right) & =\sum_{i} f_{i}\left(X_{t^{-}}^{1}, . ., X_{t^{-}}^{n}\right) d X_{t}^{i}+\sum_{i, j} \frac{1}{2} f_{i j}\left(X_{t^{-}}^{1}, . ., X_{t^{-}}^{n}\right) d\left[X^{i}, X^{j}\right]_{t}^{c} \\
& +f\left(X_{t}^{1}, . ., X_{t}^{n}\right)-f\left(X_{t^{-}}^{1}, . ., X_{t^{-}}^{n}\right)-\sum_{i} f_{i}\left(X_{t^{-}}^{1}, . ., X_{t^{-}}^{n}\right) \Delta X_{t}^{i}
\end{aligned}
$$

with $f_{i}=\frac{\partial f}{\partial x_{i}}, f_{i j}=\frac{\partial^{2} f}{\partial x_{i} x_{j}}$ and $\left[X^{i}, X^{j}\right]^{c}$ the continuous part of the mutual variation $^{8}$ of $X^{i}$ and $X^{j}$.

Now with the above results we study the solution of equation (2):

$$
d P(t)=P\left(t^{-}\right)\left[\rho_{t} d t+\sigma_{t} d Y(t)\right]=\left(a \sigma_{t}+\rho_{t}\right) P_{t^{-}} d t+\sigma_{t} P_{t^{-}}\left(c d W_{t}+d M_{t}\right)
$$

\footnotetext{
${ }^{8}$ For more details see Shiryaev (1999).
} 
When the coefficients $\rho_{t}$ and $\sigma_{t}$ are deterministic continuous function the solution of this equation is given by the Doléans-Dade exponential: ${ }^{9}$

$$
P_{t}=P_{0} \exp \left\{\int_{0}^{t} \sigma_{s} d Y_{s}+\int_{0}^{t}\left(\rho_{s}-\frac{c^{2} \sigma_{s}^{2}}{2}\right) d s\right\} \prod_{0<s \leq t}\left(1+\sigma_{s} \Delta Y_{s}\right) e^{-\sigma_{s} \Delta Y_{s}}
$$

with (13) we obtain:

$$
\begin{aligned}
P_{t}=P_{0} \quad \exp & \left\{\int_{0}^{t} c \sigma_{s} d W_{s}+\int_{0}^{t} c \sigma_{s} d M_{s}+\int_{0}^{t}\left(a \sigma_{s} \rho_{s}-\frac{c^{2} \sigma_{s}^{2}}{2}\right) d s\right\} \\
& \prod_{0<s \leq t}\left(1+\sigma_{s} \Delta M_{s}\right) e^{-\sigma_{s} \Delta M_{s}}
\end{aligned}
$$

to ensure that $P_{t} \geq 0, a . s . \forall t \in[0, T]$, we need that

$$
1+\sigma_{t} \Delta M_{t} \geq 0, \forall t \in[0, T]
$$

If we assume the convention ' $\sigma>0$ ', we only need that the jumps of $N_{t}$ be bounded from below ${ }^{10}$, i.e., $\Delta N_{t} \geq-\frac{1}{\sigma_{t}}$, it means that we consider only "semi-fat tailed" distributions as Poisson, Gamma, Hyperbolic and Normal Inverse Gaussian and we eliminate processes with heavy tails, it is worth noting that the stable distributions (without including the Gaussian Case) were eliminated when we supposed that $Y$ has all moments finite.

The following step consist in characterize all the measures that are absolutely continuous with respect to $\mathbf{P}$, to this end let:

$$
\mathcal{M}(d t, d x)=L(d t, d x)-d t G(d x)
$$

then

$$
M_{t}=\int_{0}^{t} \int_{\mathbb{R}} x \mathcal{M}(d s, d x)
$$

\footnotetext{
${ }^{9}$ See Jacod and Shiryaev (1987)

${ }^{10}$ Observe that from $N_{t}=M_{t}+a t$ we have $\Delta M_{t}=\Delta N_{t}$.
} 
Now two useful results: ${ }^{11}$

Lemma 1 Let $R_{t}$ and $K(t, x)$ be a previsible and a Borel previsible processes ${ }^{12}$ respectively. Suppose that

$$
E\left(\int_{0}^{t} R_{s}^{2} d s\right)<\infty
$$

and $K \geq 0, K(t, 0)=1 \forall t \in \mathbb{R}^{+}$. Let $k(t, x)$ be another Borel previsible process such that

$$
\int_{\mathbb{R}}[K(t, x)-1-k(t, x)] G(d x)<\infty
$$

Define a process $\mathcal{Z}_{t}$ by

$$
\begin{aligned}
\mathcal{Z}_{t} & =\exp \int_{0}^{t} R_{s} d W_{s}-\frac{1}{2} \int_{0}^{t} R_{s}^{2} d s+\int_{0}^{t} \int_{\mathbb{R}} k(s, x) \mathcal{M}(d s, d x) \\
& -\int_{[0, t) \times \mathbb{R}}[K(s, x)-1-k(s, x)] G(d x) d s \prod_{0<s \leq t} K\left(s, \Delta N_{s}\right) e^{-k\left(s, \Delta N_{s}\right)}
\end{aligned}
$$

then $\mathcal{Z}$ is a local martingale with $\mathcal{Z}_{0}=1$ and $\mathcal{Z}$ is positive if and only if $K>0$.

Proof From the fact

$$
\mathcal{Z}_{t}-\mathcal{Z}_{t^{-}}=\mathcal{Z}_{t^{-}}\left(K\left(t, \Delta N_{t}\right)-1\right)
$$

and applying Itô's formula and (14), we can obtain:

$$
\mathcal{Z}_{t}=1+\int_{0}^{t} R_{s} \mathcal{Z}_{s}-d W s+\int_{0}^{t} \int_{\mathbb{R}} \mathcal{Z}_{s}-[K(s, x)-1] \mathcal{M}(d s, d x)
$$

\footnotetext{
${ }^{11}$ See Chan (1999)

${ }^{12}$ a process $K_{\omega}(t, x)$ is said to be a Borel previsible function or process if the process $t \mapsto$ $K_{\omega}(t, x)$ is a previsible function for fixed $x$ and the function $x \mapsto K_{\omega}(t, x)$ is Borel-measurable for fixed $t$. 
This expression is a local martingale.

Teorem $\mathbf{1}$ Let $\mathbf{Q}$ be a measure which is absolutely continuous with respect to $\mathbf{P}$ on $\mathcal{F}_{T}$. Then

$$
\left.\frac{d \mathbf{Q}}{d \mathbf{P}}\right|_{\mathcal{F}_{T}}=\mathcal{Z}_{T}
$$

where $\mathcal{Z}$ is as in the lemma 1 , for some $R, K$ and $k$ for which $E \mathcal{Z}_{T}=1$. Moreover under $\mathbf{Q}$, the process

$$
\hat{W}_{t}=W_{t}-\int_{0}^{t} R_{s} d s
$$

is a Brownian Motion and the process $N_{t}$ is a quadratic pure jump process with compensator measure given by $d t \hat{G}_{t}(d x)$ with

$$
\hat{G}_{t}(d x)=K(t, x) G(d x)
$$

and constant part given by

$$
\hat{a}_{t}=E^{\mathbf{Q}} N_{t}=a t+\int_{0}^{t} \int_{\mathbb{R}} x(K(s, t)-1) G(d x) d s
$$

Then under $\mathbf{Q}$ the process $N_{t}$ can be represented as

$$
N_{t}=\hat{M}_{t}+a t+\int_{0}^{t} \int_{\mathbb{R}} x(K(s, t)-1) G(d x) d s
$$

with

$$
\hat{M}_{t}=M_{t}-\int_{0}^{t} \int_{\mathbb{R}} x(K(s, t)-1) G(d x) d s
$$
et

This process is a $\mathbf{Q}$-martingale and it is easy to see that $\Delta \hat{M}_{t}=\Delta M_{t}$. Now

$$
\hat{P}_{t}=\exp \left(-\int_{0}^{t} r_{s} d s\right) P_{t}
$$


be the discounted price process. Replacing the processes $W_{t}$ and $M_{t}$ in the equation (15) by their respective $\mathbf{Q}$-versions, we obtain

$$
\begin{aligned}
\hat{P}_{t} & =P_{0} \exp \int_{0}^{t} c \sigma_{s} d \hat{W}_{s}+\int_{0}^{t} c \sigma_{s} d \hat{M}_{s}+\int_{0}^{t}\left(a \sigma_{s}+c \sigma_{s} R_{s}+\rho_{s}-r_{s}-\frac{c^{2} \sigma_{s}^{2}}{2}\right) d s \\
& +\int_{0}^{t} \sigma_{s} \int_{\mathbb{R}} x(K(s, x)-1) G(d x) d s \prod_{0<s \leq t}\left(1+\sigma_{s} \Delta \hat{M}_{s}\right) e^{-\sigma_{s} \Delta \hat{M}_{s}}
\end{aligned}
$$

A necessary and sufficient condition for $\hat{P}_{t}$ be a $\mathbf{Q}-$ martingale is the existence of $R$ and $K>0$ a.s. for which:

$$
c R_{s}+a+\frac{\rho_{s}-r_{s}}{\sigma_{s}}+\int_{\mathbb{R}} x(K(s, x)-1) G(d x)=0 \forall s
$$

and $E \mathcal{Z}_{t}=1, \forall t>0$. Since the process

$$
\exp \left\{\int_{0}^{t} c \sigma_{s} d \hat{W}_{s}+\int_{0}^{t} \sigma_{s} d \hat{M}_{s}-\int_{0}^{t} \frac{c^{2} \sigma_{s}^{2}}{2} d s\right\} \prod_{0<s \leq t}\left(1+\sigma_{s} \Delta \hat{M}_{s}\right) e^{-\sigma_{s} \Delta \hat{M}_{s}}
$$

is a $\mathbf{Q}$-martingale. Now we can state the following

Theorem 2 If there exist $R$ and $K>0$ for which $E \mathcal{Z}_{t}=1 \forall t$ and the market price of risk is given by

$$
\eta(s)=\frac{\rho_{s}-r_{s}}{\sigma_{s}}=\int_{\mathbb{R}} x(1-K(s, x)) G(d x)-c R_{s}-a \forall s
$$

Then the market $\mathfrak{M}$ is arbitrage free.

Proof Take a tame portfolio $\pi$ and suppose that $\mathbf{P}\left(M^{\pi}(T) \geq 0\right)=1$, by definition we have

$$
M^{\pi}(t)=\int_{0}^{t} \gamma(s) \pi(s)[\sigma(s) d Y(s)+(\rho(s)-r(s)) d s]
$$


With equation (13) and (19) we obtain

$$
\begin{aligned}
M^{\pi}(t) & =\int_{0}^{t} \gamma(s) \pi(s) \sigma(s)\left[c d W(s)+d M(s)+\left(\int_{\mathbb{R}} x(1-K(s, x)) G(d x)\right.\right. \\
& \left.\left.\left.-c R_{s}\right) d s\right)\right]
\end{aligned}
$$

Now using (16) and (17)

$$
M^{\pi}(t)=\int_{0}^{t} \gamma(s) \pi(s) \sigma(s)[c d \hat{W}(s)+d \hat{M}(s)]
$$

We have that $M^{\pi}$ is a $\mathbf{Q}$ - local martingale bounded from below, since $\pi$ is tame, then a supermartingale. Hence

$$
E^{\mathbf{Q}} M^{\pi}(T) \leq 0
$$

then $\mathbf{Q}\left(M^{\pi}(T)>0\right)=0$, so there are not arbitrage opportunities.

From these result we know that our market is arbitrage free. Now we can introduce the investor problem.

\section{Optimization Problem}

In this section we formalize the individual problem of the investor, give some definitions and finally we present the main result.

Definition 4 A pair $(\pi, C)$ of portfolio consumption process is called admissible for the initial capital $x \geq 0$. If

$$
X(T)=X^{x, \pi, C}(T) \geq 0, \text { a.s. }
$$

The class of all such pairs will be denoted by $\mathcal{A}(x)$.

Now take any equivalent martingale measure $\mathbf{Q}$ and define the following state price density:

$$
H^{\mathcal{Z}}(t)=\gamma(t) \mathcal{Z}(t), \forall t \in[0, T]
$$

where 


$$
\left.\frac{d \mathbf{Q}}{d \mathbf{P}}\right|_{\mathcal{F}_{t}}=\mathcal{Z}_{t}
$$

using (5) and the generalized Itô's lemma for $F(\mathcal{Z}(t), \gamma(t) X(t))=H^{\mathcal{Z}}(t) X(t)$, we obtain:

$$
\begin{array}{ll}
d & \left(H^{\mathcal{Z}}(t) X(t)\right)=-H^{\mathcal{Z}}\left(t^{-}\right) d C(t)+H^{\mathcal{Z}}\left(t^{-}\right) \pi(t)(\rho(t)-r(t)) d t \\
+ & H^{\mathcal{Z}}\left(t^{-}\right) \pi(t) \sigma(t) d Y(t)+\gamma(t) X\left(t^{-}\right) d \mathcal{Z}(t)+H^{\mathcal{Z}}(t) \Delta X(t)
\end{array}
$$

Now with the decomposition (13), we obtain

$$
\begin{aligned}
d\left(H^{\mathcal{Z}}(t) X(t)\right) & =-H^{\mathcal{Z}}\left(t^{-}\right) d C(t)+H^{\mathcal{Z}}\left(t^{-}\right) \pi(t) \sigma(t)[c d W(t)+d M(t)] \\
& +\gamma(t) X\left(t^{-}\right) d \mathcal{Z}(t)+d D(t)
\end{aligned}
$$

where $D(t)$ is given by

$$
D(t)=D(0)+\int_{0}^{t} H^{\mathcal{Z}}\left(t^{-}\right) \pi(t)(\sigma(t) a+\rho(t)-r(t)) d t+\sum_{0<s \leq t} H^{\mathcal{Z}}(s) \Delta X(s)
$$

In order to formulate our optimization problem we need the concept of utility function.

Definition 5 We say that a function $u:(0, \infty) \rightarrow R$ is a utility function if it is strictly increasing, strictly concave, continuously differentiable and

$$
u^{\prime}(\infty) \triangleq \lim _{x \rightarrow \infty} u^{\prime}(x)=0 \text { and } u^{\prime}\left(0_{+}\right) \triangleq \lim _{x \downarrow 0} u^{\prime}(x)=\infty
$$

Examples of utility function are $u(x)=\log x$ and $u(x)=\frac{x^{\delta}}{\delta}, \delta \in(-\infty, 1) 0$.

We will denote by $I(\cdot)$ the inverse of the derivative $u^{\prime}(\cdot)$, both these functions are continuous, strictly decreasing, and map $(0, \infty)$ onto itself with $I\left(0_{+}\right)=$ $u^{\prime}\left(0_{+}\right)=\infty, I(\infty)=u^{\prime}(\infty)=0$. We shall consider also de convex dual

$$
\widehat{u}(y) \triangleq \max _{0<x<\infty}[u(x)-x y]=u(I(y))-y I(y), 0<y<\infty
$$


Of $u(\cdot)$ : a convex decreasing function, continuously differentiable on $(0, \infty)$ and satisfies

$$
\begin{gathered}
\widehat{u}^{\prime}(y)=-I(y), 0<y<\infty \\
u(x) \triangleq \min _{0<y<\infty}[\widehat{u}(y)+x y]=\widehat{u}\left(u^{\prime}(x)\right)+x u^{\prime}(x) \\
\widehat{u}(\infty)=u\left(0_{+}\right), \widehat{u}\left(0_{+}\right)=u(\infty)
\end{gathered}
$$

\section{Remarks on Utility Functions}

- Observe that (23) is the usual Inada's condition.

- Negative wealth is not allowed to consider utilities that are economically relevant, as is the case of the $u(x)=\ln (x)$. But, we could take $u(0)=-\infty$ and make $u:[0, \infty) \rightarrow R \bigcup\{-\infty\}$

- In general we must have that marginal utility from consumption tends to infinite when wealth tends to the infimum of its allowed values.

Now consider an small investor who has an initial capital $x>0$, and he wants to choose a portfolio $\pi(\cdot)$ and consumption processes $\{c(t), 0 \leq t \leq T\}$ in order to maximize his expected utility from the terminal wealth $X^{x, \pi, C}(T)$ and from consumption.

Given the utility functions $g$ and $u(t, \cdot)$, as in the above definition, we define the following classes:

$$
\begin{gathered}
\mathcal{A}_{(x)} \triangleq\left\{(\pi, C) \in \mathcal{A}(x) / E g^{-}\left(X^{x, \pi, C}(T)\right)<\infty\right\} \\
\mathcal{A}_{u}(x) \triangleq\left\{(\pi, C) \in \mathcal{A}(x) / E \int_{0}^{T} u^{-}(t, c(t)) d t<\infty\right\}
\end{gathered}
$$

remember $f^{-}(x)=\max -f(x), 0$.

Then our small investor will have to maximize the expected utility from consumption and terminal wealth over the following class:

$$
\mathcal{A}_{0}(x) \triangleq \mathcal{A}_{u}(x) \cap \mathcal{A}_{g}(x)
$$


The value function will be

$$
V_{\mathcal{Z}}(x)=\sup _{(\pi, C) \in \mathcal{A}_{0}(x)} E\left[\int_{0}^{T} u(t, c(t)) d t+g\left(X^{x, \pi, C}(T)\right)\right]
$$

Now to solve this optimization problem consider, in the context of the market model $\mathfrak{M}$ described before, a contingent claim ${ }^{13} \xi$ and a consumption process $C$ that satisfy

$$
E\left[H^{\mathcal{Z}}(T) \xi+\int_{0}^{T} H^{\mathcal{Z}}\left(t^{-}\right) d C(t)\right]=x>0
$$

Then if there exist a portfolio process $\pi(\cdot)$, such that $(\pi, C) \in \mathcal{A}(x)$ and $X^{x, \pi, C}(T)=\xi$; we could conclude that the optimal problem is in some sense equivalent to the following problem:

$$
\max E\left[\int_{0}^{T} u(t, c(t)) d t+g(\xi)\right]
$$

over all pairs $(\xi, c)$ of contingent claims and consumption rate process that satisfy the constraint $(29)$.

Now with $y>0$ (Lagrange multiplier) and with (24):

$$
\begin{array}{r}
E\left[\int_{0}^{T} u(t, c(t)) d t+g(\xi)\right]+y\left[x-E\left[H^{\mathcal{Z}}(T) \xi+\int_{0}^{T} H^{\mathcal{Z}}\left(t^{-}\right) c(t) d t\right]\right]= \\
=E\left[\int_{0}^{T}\left[u(t, c(t)) d t-y H^{\mathcal{Z}}\left(t^{-}\right) c(t)\right] d t\right]+E\left[g(\xi)-y H^{\mathcal{Z}}(T) \xi\right]+x y \\
\leq E\left[\int_{0}^{T} \widehat{u}\left(t, y H^{\mathcal{Z}}\left(t^{-}\right)\right) d t\right]+E\left[\widehat{g}\left(y H^{\mathcal{Z}}(T)\right)\right]+x y
\end{array}
$$

the equality holds if and only if

$$
\xi_{\mathcal{Z}}=I_{g}\left(y H^{\mathcal{Z}}(T)\right) \text { and } c_{\mathcal{Z}}(t)=I_{u}\left(t, y H^{\mathcal{Z}}\left(t^{-}\right)\right)
$$

\footnotetext{
${ }^{13} \mathrm{~A}$ contingent claim $\xi$ is a random variable $\mathcal{F}_{T}$-measurable.
} 
then in the constraint (29), we define

$$
\mathcal{X}_{\mathcal{Z}}(y) \triangleq E\left[H^{\mathcal{Z}}(T) I_{g}\left(y H^{\mathcal{Z}}(T)\right)+\int_{0}^{T} H^{\mathcal{Z}}\left(t^{-}\right) I_{u}\left(t, y H^{\mathcal{Z}}\left(t^{-}\right)\right) d t\right]=x
$$

and if we consider $x \in(0, \infty)$ that is $\mathcal{X}_{\mathcal{Z}}(y)<\infty, \forall 0<y<\infty$. This function maps $(0, \infty)$ onto itself and is continuous, strictly decreasing with

$$
\mathcal{X}_{\mathcal{Z}}\left(0_{+}\right) \triangleq \lim _{y \downarrow 0} \mathcal{X}_{\mathcal{Z}}(y)=\infty, \mathcal{X}_{\mathcal{Z}}(\infty) \triangleq \lim _{y \rightarrow \infty} \mathcal{X}_{\mathcal{Z}}(y)=0
$$

If we denote by $\mathcal{Y}^{\mathcal{Z}}(\cdot)=\mathcal{X}_{\mathcal{Z}}^{-1}(\cdot)$. Then the lagrange multiplier $y>0$ is uniquely determined by

$$
y=\mathcal{Y}^{\mathcal{Z}}(x)
$$

Now we can state the main result

Theorem 3 Suppose $x \in(0, \infty)$ and $V_{\mathcal{Z}}(x)<\infty, \forall x \in(0, \infty)$ For any $x>0$, consider the optimization problem with value function $V_{\mathcal{Z}}(x)$ as in (??) and define $\xi_{\mathcal{Z}}$ and $c_{\mathcal{Z}}(\cdot)$ as in (31). Then if

a) there is a portfolio process $\pi_{\mathcal{Z}}(\cdot)$ such that $\left(\pi_{\mathcal{Z}}, C_{\mathcal{Z}}\right) \in \mathcal{A}(x)$ and

$$
X^{x, \pi_{\mathcal{Z}}, C_{\mathcal{Z}}}(T)=\xi_{\mathcal{Z}}
$$

b) the process $D_{t}$ is a Local martingale and $D_{0}=0$.

Then $\left(\pi_{\mathcal{Z}}, c_{\mathcal{Z}}\right)$ are the solutions of the optimal problem and the value function is given by

$$
V_{\mathcal{Z}}(x)=\mathcal{G}\left(\mathcal{Y}_{\mathcal{Z}}(x)\right)
$$

where

$$
\mathcal{G}(y) \triangleq E\left[\int_{0}^{T} u\left(t, I_{u}\left(t, y H^{\mathcal{Z}}\left(t^{-}\right)\right) d t+g\left(I_{g}\left(y H^{\mathcal{Z}}(T)\right)\right)\right], \forall y \in(0, \infty)\right.
$$


and the convex dual of $V_{\mathcal{Z}}(\cdot)$ is

$$
\widehat{V_{\mathcal{Z}}}(y)=\mathcal{G}(y)-y \mathcal{X}_{\mathcal{Z}}(y)=E\left[\int_{0}^{T} \widehat{u}\left(t, y H^{\mathcal{Z}}\left(t^{-}\right)\right) d t\right]+E\left[\widehat{g}\left(y H^{\mathcal{Z}}(T)\right)\right]
$$

Proof By construction $\xi_{\mathcal{Z}}$ and $c_{\mathcal{Z}}$ satisfy (29) and using the following inequality

$$
f\left(I_{f}(y)\right) \geq f(x)+y\left[I_{f}(y)-x\right]
$$

for every utility function $f$, we obtain

$$
\begin{aligned}
u\left(t, c_{\mathcal{Z}}(t)\right) \geq & \geq(t, 1)+\mathcal{Y}_{\mathcal{Z}}(x) H^{\mathcal{Z}}\left(t^{-}\right)\left(c_{\mathcal{Z}}(t)-1\right), 0 \leq t \leq T \\
& g \quad\left(\xi_{\mathcal{Z}}\right) \geq g(1)+\mathcal{Y}_{\mathcal{Z}}(x) H^{\mathcal{Z}}(T)\left(\xi_{\mathcal{Z}}-1\right), \text { a.s. }
\end{aligned}
$$

therefore

$$
\begin{aligned}
& E \quad\left[\int_{0}^{T} u^{-}\left(t, c_{\mathcal{Z}}(t)\right) d t+g^{-}\left(\xi_{\mathcal{Z}}\right)\right] \leq|g(1)|+\int_{0}^{T}|u(t, 1)| d t \\
& +\mathcal{Y}_{\mathcal{Z}}(x)\left[H^{\mathcal{Z}}(T)+\int_{0}^{T} H^{\mathcal{Z}}\left(t^{-}\right) d t\right]<\infty
\end{aligned}
$$

Since $E H^{\mathcal{Z}}(t) \leq e^{r T}, 0 \leq t \leq T$, where $r=\sup _{0 \leq t \leq T} r(t)<\infty$ and by (a) we have that there exist a portfolio process $\pi_{\mathcal{Z}}$ with $\left(\pi_{\mathcal{Z}}, C_{\mathcal{Z}}\right) \in \mathcal{A}(x)$ (also in $\mathcal{A}_{0}(x)$ thanks to (34)) and $X^{x, \pi_{\mathcal{Z}}, C_{\mathcal{Z}}}(T)=\xi_{\mathcal{Z}}$ a.s.).

Now take an arbitrary $x>0,(\pi, C) \in \mathcal{A}_{0}(x)$ and $y>0$ from (b) we have that the following process is a bounded (from below) local martingale:

$$
H^{\mathcal{Z}}(t) X(t)+\int_{0}^{t} H^{\mathcal{Z}}\left(s^{-}\right) d C(s)
$$

then a supermartingale and with (30) we have 


$$
\begin{gathered}
E\left[\int_{0}^{T} u(t, c(t)) d t+g\left(X^{x, \pi, C}(T)\right)\right] \leq E\left[\int_{0}^{T} u(t, c(t)) d t+g\left(X^{x, \pi, C}(T)\right)\right]+(34) \\
+y\left[x-E\left[H^{\mathcal{Z}}(T) X^{x, \pi, C}(T)+\int_{0}^{T} H^{\mathcal{Z}}\left(t^{-}\right) d C(t)\right]\right] \leq Q(y)+x y
\end{gathered}
$$

where

$$
Q(y) \triangleq E\left[\int_{0}^{T} \widehat{u}\left(t, y H^{\mathcal{Z}}\left(t^{-}\right)\right) d t+\widehat{g}\left(y H^{\mathcal{Z}}(T)\right)\right]=\mathcal{G}(y)-y \mathcal{X}_{\mathcal{Z}}(y)
$$

in particular follows

$$
V_{\mathcal{Z}}(x) \leq Q(y)+x y, \forall x>0
$$

hence

$$
\widehat{V}_{\mathcal{Z}}(y) \leq Q(y), \forall y>0
$$

On the other hand the inequality (34) holds as equality if and only if $y=\mathcal{Y}_{\mathcal{Z}}(x)$ and $(\pi, c)=\left(\pi_{\mathcal{Z}}, c_{\mathcal{Z}}\right)$ then

$$
\begin{aligned}
& E\left[\int_{0}^{T} u\left(t, c_{\mathcal{Z}}(t)\right) d t+g\left(X^{x, \pi_{\mathcal{Z}}, c_{\mathcal{Z}}}(T)\right)\right]=Q\left(\mathcal{Y}_{\mathcal{Z}}(x)\right)+x \mathcal{Y}_{\mathcal{Z}}(x) \\
& =\mathcal{G}\left(\mathcal{Y}_{\mathcal{Z}}(x)\right)
\end{aligned}
$$

now

$$
V_{\mathcal{Z}}(x)=\mathcal{G}\left(\mathcal{Y}_{\mathcal{Z}}(x)\right)
$$

and also

$$
Q(y)=V_{\mathcal{Z}}\left(\mathcal{X}_{\mathcal{Z}}(y)\right)-y \mathcal{X}_{\mathcal{Z}}(y) \leq \sup _{x>0}\left[V_{\mathcal{Z}}(x)-x y\right]
$$

For every $y>0$, and in conjunction with (35) we obtain $Q(y)=\widehat{V}_{\mathcal{Z}}(y), \forall y>0$. 


\section{Remarks on the Assumptions}

- Assumption (a) is necessary and sufficient for the optimality of consumption. For the existence of the portfolio financing this optimal consumption we need to solve the following equation:

$$
\begin{aligned}
\gamma(T) I_{g}\left(y H^{\mathcal{Z}}(T)\right) & =x-\int_{0}^{T} \gamma(t) d C_{\mathcal{Z}}(t) \\
& +\int_{0}^{T} \gamma(t) \pi_{\mathcal{Z}}(t)[\sigma(t) d Y(t)+(\rho(t)-r(t)) d t]
\end{aligned}
$$

- Assumption (b) is a sufficient condition which allow us to control the jumps on wealth and depending on the process $Y$ considered, it can be easy verified as we will see in section 6 with an example. Condition $D_{0}=0$ is just to simplify the proof. We can prove the same with $D_{0} \neq 0$.

As we have seen in section 3, we obtained a characterization of the EMM. But it can be argued that choosing an EMM can be a hard task to solve, for these reason we show how to choose an EMM in the following section.

\section{Choosing a Measure}

There exists some approaches to choose one EMM, we concentrate our attention in the approach introduced by Gerber and Shiu (1994), define the new probability

$$
\frac{d \mathbf{P}_{t}^{\theta}}{d \mathbf{P}_{t}}=\mathcal{Z}^{\theta}(t)=e^{\theta Y_{t}-t \log \varphi(\theta)}
$$

where $\varphi(\theta)=E e^{\theta Y_{1}}$. When the stock price process has constant coefficients, Gerber and Shiu (1994) prove that for a given constant $r$ it is possible to find a solution $\theta$ of the following:

$$
r=\log \left(\frac{\varphi(\theta+1)}{\varphi(\theta)}\right)
$$


Then we can verify that the process $\hat{P}_{t}=e^{-r t} P_{t}$ is a martingale under $\mathbf{P}^{\theta}$,i.e. $\mathbf{P}^{\theta} \in \mathcal{Q}$ (set of EMM). Moreover the process is a Lévy Process under this probability and is called the Esscher transform ${ }^{14}$ of the original process. In our model we consider time dependent functions, then we consider the generalized Esscher Transform:

$$
\frac{d \mathbf{P}_{t}^{\theta}}{d \mathbf{P}_{t}}=\mathcal{Z}^{\theta}(t)=e^{\left\{\int_{0}^{t} \theta_{s} d Y_{s}-\int_{0}^{t} \log \varphi\left(\theta_{s}\right) d s\right\}}
$$

Now we can choose $\theta_{s}$ in order to satisfy equation (18), since this is the case of $K(s, x)=\exp \left(-\theta_{s} x\right), k(s, x)=-\theta_{s} x$ and $R_{s}=-c \theta_{s}$. In fact with these expressions we obtain

$$
-c^{2} \sigma_{s} \theta_{s}+a \sigma_{s}+\rho_{s}-r_{s}+\sigma_{s} \int_{\mathbb{R}} x\left(e^{-\theta_{s} x}-1\right) G(d x)=0
$$

It easy to verify that this equation has an unique solution for which $\varphi\left(\theta_{s}\right)<\infty$ and $\theta_{s} \in\left(-b_{1}, b_{2}\right) \forall s$. Then we can get a EMM, and important fact of this measure is that this is the measure of minimum relative entropy with respect to $\mathbf{P}$, to see this remember the definition of entropy:

$$
I_{\mathbf{P}}(\mathbf{Q})=E^{\mathbf{Q}}\left[\log \frac{d \mathbf{Q}}{d \mathbf{P}}\right]
$$

Where $\mathbf{Q}$ is any absolutely continuous measure with respect to $\mathbf{P}$, with lemma 1 we have

$$
I_{\mathbf{P}}(\mathbf{Q})=E^{\mathbf{Q}}\left[\frac{1}{2} \int_{0}^{T} R_{s}^{2} d s+\int_{0}^{T} \int_{\mathbb{R}}[K(s, x)(\log K(s, x)-1)+1] G(d x) d s\right]
$$

where $\mathbf{Q}$ depends on the choice of $K$ and $R$, and these functions have to satisfy equation (18). We can show ${ }^{15}$ that this minimum is obtained when $K=$ $\exp (-x \sigma \lambda)$ and $R=-c \sigma \lambda$, where $\lambda$ is the lagrange multiplier associated to the constraint (18), this can justify the choice of the measure associated to $\theta=\sigma \lambda$.

\footnotetext{
${ }^{14}$ For more details on Esscher transforms see Buhlmann et al. (1996)

${ }^{15}$ See Chan (1999)
} 


\section{Example}

Take $\sigma=\rho=c=1$ and $r=0$. Now assume that $N_{t}=\frac{N_{1}(t)-N_{2}(t)}{2}$, where $N_{i}$ is a Poisson process with rate 1 , so:

- $G=\delta_{-\frac{1}{2}}+\delta_{\frac{1}{2}}$

- $a=E N_{t}=0$

Now we choose $k(x)=\frac{2 x}{3}$ and $K(x)=1-k(x)$, replacing in condition (18) we obtain $R_{s}=-\frac{2}{3}$, from the fact that $\Delta N_{s}=0$, we have

$$
\mathcal{Z}_{t}=\exp \left\{-\frac{2}{3} W_{t}-\frac{2 t}{9}+\frac{2}{3} N_{t}\right\}
$$

It is easy to verify that $E \mathcal{Z}_{T}=1$, in other words it is a EMM density. So we have find one EMM. Now we apply the approach presented in last section to find the EMM that minimize entropy: as $\sigma=1$, then $\theta=\lambda$. We obtain the parameter $\theta$ that satisfy equation (38):

$$
-\theta+1+\int x\left(e^{-\theta x}-1\right)\left(\delta_{-\frac{1}{2}}+\delta_{\frac{1}{2}}\right)(d x)=0
$$

reducing this expression, we have:

$$
1-\theta+\frac{e^{-\frac{\theta}{2}}-e^{\frac{\theta}{2}}}{2}=0
$$

the solution of this equation is $\theta^{*} \approx 0,6626$. In equation (5):

$$
\mathcal{Z}^{\theta *}(t)=e^{\theta^{*} Y_{t}-t \log \varphi\left(\theta^{*}\right)}
$$

we have also $Y_{t}=W_{t}+N_{t}$ and

$$
\begin{aligned}
\varphi(\theta) & =E e^{\theta W_{1}+\theta N(1)} \\
& =E e^{\theta W_{1}} E e^{\theta N(1)} \\
& =E e^{\theta W_{1}} \\
& =e^{\frac{\theta^{2}}{2}}
\end{aligned}
$$

where the first equality is due to the independence of $W_{t}$ and $N(t)$, the second is due to the fact that $N_{1}(t)$ and $N_{2}(t)$ are independent and identically distributed, and the third is the expected value of a Log-normal variable. From here we have 


$$
\mathcal{Z}^{\theta *}(t)=e^{\left\{0,6626 W_{t}+0,6626 N_{t}-0,21952 t\right\}}
$$

and this measure is obtained when $K(s, x)=e^{-0,6626 x}, k(s, x)=-0,6626 x$ and $R_{s}=-0,6626$.

Now as $N_{t}$ and $W_{t}$ are martingales condition (b) in the main theorem is satisfied. Then if we take $u(t, x)=g(x)=\log (x)$, then $I_{u}(x)=I_{g}(x)=\frac{1}{x}$ and

$$
\xi_{\mathcal{Z}}=\frac{1}{y H^{\mathcal{Z}}(T)} \text { and } c_{\mathcal{Z}}(t)=\frac{1}{y H^{\mathcal{Z}}\left(t^{-}\right)}
$$

but

$$
\begin{aligned}
x=\mathcal{X}^{\mathcal{Z}}(y) & =E\left[H^{\mathcal{Z}}(T) I_{g}\left(y H^{\mathcal{Z}}(T)\right)+\int_{0}^{T} H^{\mathcal{Z}}\left(t^{-}\right) I_{u}\left(t, y H^{\mathcal{Z}}\left(t^{-}\right)\right) d t\right] \\
& =E\left[\frac{1}{y}+\frac{T}{y}\right]
\end{aligned}
$$

then $y=\frac{T+1}{x}$ and

$$
\xi_{\mathcal{Z}}=\frac{x}{(T+1) \mathcal{Z}(T)} \text { and } c_{\mathcal{Z}}(t)=\frac{x}{(T+1) \mathcal{Z}\left(t^{-}\right)}
$$

So depending on the state price density, i.e., on the EMM we choose, we have the path of the optimal policies. More precisely, if we desire to choose the EMM closest to $\mathbf{P}$ in terms of its information contents, since $\mathbf{P}$ contains information about the behavior of the market, we have to choose the EMM given by (39). ${ }^{16}$

\section{Conclusions}

In this paper we studied the optimal investment and consumption problem assuming that the risky asset is driven by a geometric Lévy process which allow us to obtain more realistic results. Another important issue addressed in this paper is the characterization of the EMM in a Lévy market and how to choose one of them using a minimal entropy criteria.

An important and difficult issue not considered in the paper is the availability of the portfolio processes, since many of the market models considered in the paper

\footnotetext{
${ }^{16}$ For more details on how to construct EMM with different types of Lévy processes see Fajardo (2003).
} 
can be incomplete, we need to ensure that the financing portfolio is available in the market. For a discussion on this type of problem see Cvitanić and Karatzas (1992). Also from the existence of EMM we obtained the arbitrage free economies, with this in mind some equilibrium analysis in continuous time can be done.

\section{References}

Benth, F. E., Karlsen, K. H., \& Reikvam, K, . (2001). Optimal portfolio selection with consumption and nonlinear integro-differential equations with gradient constraints: A viscosity solution approach. Finance and Stochastics, (5):275303 .

Buhlmann, H., Delbaen, F., Embrechts, P., \& Shirjaev, A. (1996). No-arbitrage, change of measure and conditional esscher transforms. CWI Quaterly, (9):291317 .

Chan, T. (1999). Pricing contingent claims on stocks driven by Lévy processes. Annals of Applied Probability, 9(2):504-528.

Cvitanić, J. \& Karatzas, I. (1992). Convex duality in constrained portfolio optimization. Annals of Applied Probability, (2):767-818.

Eberlein, E. \& Jacod, J. (1997). Hyperbolic distributions in finance. Finance and Stochastics, (1):281-299.

Fajardo, J. (2000). Optimal consumption and investment with hyperbolic Lévy motion. Brazilian Review of Econometrics, 20(1):27-54.

Fajardo, J. (2003). Equivalent martingale measures and Lévy processes. Working paper IBMEC.

Gerber, H. \& Shiu, E. (1994). Option pricing by esscher-transform. Transactions of the Society of Actuaries, 46:99-191.

Jacod, J. \& Shiryaev, A. N. (1987). Limit Theorems for Stochastic Processes. Springer-Verlag, New York.

Karatzas, I. \& Shreve, S. E. (1998). Methods of Mathematical Finance. SpringerVerlag, New York. 
Kramkov, D. O. \& Schachermayer, W. (1999). The asymptotic elasticity of utility functions and optimal investment in incomplete markets. Annals of Applied Probability, 11(3):904-950.

Merton, R. C. (1971). Optimum consumption and portfolio rules in a continuoustime model. Journal of Economic Theory, (3):373-413.

Rydberg, T. (1997). Why financial data are interesting to statisticians. Centre for Analytical Finance, Aarhus University. Working Paper 5.

Sato, K.-I. (1999). Lévy Processes and Infinitely Divisible Distributions. Cambridge University Press, Cambridge.

Shiryaev, A. N. (1999). Essential of Stochastic Finance: Facts, Models and Theory. World Scientific Pub. Co. 